\title{
Current Rectification with Poly---Lysine-Coated Quartz Nanopipettes
}

\author{
Senkei Umehara $\dagger, \ddagger$, Nader Pourmand $\dagger$, Chris D. Webb§, Ronald W. Davis $\dagger$, Kenji \\ Yasuda $\dagger, \ddagger$, and Miloslav Karhanek ${ }^{\star}, \dagger$ \\ tStanford Genome Technology Center, Department of Biochemistry, Stanford University, 855 \\ California Avenue, Palo Alto, California 94304 \\ ‡Department of Life Sciences, Graduate School of Arts and Sciences, The University of Tokyo, 3-8-1 \\ Komaba, Meguro, Tokyo 153-8902 Japan \\ $\S$ Stanford University School of Medicine, Stanford University, 855 California Avenue, Palo Alto, \\ California 94304
}

\begin{abstract}
Ion current rectification with quartz nanopipette electrodes was investigated through the control of the surface charge. The presence and absence of a positively charged poly--Llysine (PLL) coating resulted in the rectified current with opposite polarity. The results agreed with the theories developed for current-rectifying conical nanopores, suggesting the similar underlying mechanism among asymmetric nanostructure in general. This surface condition dependence can be used as the fundamental principle of multi-purpose real-time in vivo biosensors.
\end{abstract}

Nanomaterials are being widely exploited by recent technologies because of their extraordinary properties. Although the development of fabrication processes for particular nanostructures poses great challenges by itself, the practical use of these nanomaterials is also of great interest for biology and medicine. ${ }^{1}$ Because of their structural diversity, these materials are often categorized and referred to as nanoparticles, ${ }^{2}$ nanowires, ${ }^{3}$ nanotubes, ${ }^{4}$ nanopores, ${ }^{5}$ or nanopatterned surfaces. ${ }^{6}$ Nanopipettes are among these; a nanopipette is defined as a pipette with a very fine tip that has a nanoscale opening. Nanolithography is one of the typical applications of nanopipettes as a delivery tool of a tiny amount of chemicals. ${ }^{7,8}$

Nanopipettes are versatile enough to be used as a tool for sensitive detection in biomedical applications. Optical detection of fluorescently labeled macromolecules such as DNA or proteins with nanopipettes has been reported. ${ }^{9,10}$ Fully electrical detection has also been shown with similarly sized nanoparticles, whose flow through the nanopipette opening creates temporal current blockades. ${ }^{11}$ The ultimate goal of these efforts, the label-free real-time electrical detection of single molecules, could be achieved eventually by a deeper understanding of the fundamental characteristics of nanopipette electrodes under an external electric field. It not only helps to unveil the dynamics of biological systems but can also have a remarkable impact on drug screening and pathogen detection.

(C) 2006 American Chemical Society

*Corresponding author. karhanek@stanford.edu. Tel: +1 650812 1960. Fax: +1 6508121975.

Supporting Information Available: Experimental details and procedures regarding nanopipette fabrication, nanopipette coating, electrical measurement, data analysis, and estimation of the effective length as a sensing zone. This material is available free of charge via the Internet at http://pubs.acs.org. 
Interestingly, although a general understanding of nanopipette electrodes can be based on the understanding of microelectrodes, the unique nanoscale geometry often causes characteristic behavior that requires further focused studies. For example, related studies have examined the physicochemical properties of nanopipettes under varying conditions such as electrolyte concentrations and $\mathrm{pH},{ }^{12}$ or polyethylene glycol polymer coatings. ${ }^{13}$ Similarly shaped goldplated conical nanopores have been studied in a more detailed manner, involving observations of the role of surface charge in the ionic current. ${ }^{14}$

Our target here is the effect of cationic polymer coating on a glass nanopipette surface, providing the basis for functionalized nanopipettes that will be used as sensitive biosensors. By understanding how ions flow through the nanometer-sized opening, how these ions interact with the surface inside and outside the tip, and what happens if the surface is modified by the cationic polymer coating, we can obtain practical insight to further functionalize these coated nanopipettes for specific applications. A technical challenge, however, is successful and uniform decoration of the surface of nanopipettes. For example, controlled silanization is said to be extremely difficult when the nanopipette diameter becomes smaller than $200 \mathrm{~nm} .{ }^{15} \mathrm{We}$ overcame this challenge using a hydrophilic, positively charged poly-L-lysine (PLL) polymer as a coating reagent. A stabilized PLL layer created on a nanopipette tip surface is fully functional to other amine-reactive reagents widely available.

Here we compare the fundamental characteristics of the current response between noncoated and PLL-coated nanopipette electrodes to an applied voltage and show that the current signal induced by an identical voltage input was dramatically affected by PLL coating. Noncoated quartz nanopipettes responded to an alternating sine wave voltage input by exhibiting an asymmetric output current, an effect already recognized as current rectification. ${ }^{12}$ PLL-coated nanopipettes also rectified the current; however, compared with the noncoated ones, the current was amplified and rectified in an opposite direction. In both cases, current was rectified when the applied voltage amplitude was higher than $10^{-1} \mathrm{~V}$ and the frequency was lower than $10^{1}$ $\mathrm{Hz}$, but the exact threshold values likely depend on the surface charge and specific nanopipette geometry. The contribution of surface charge was further confirmed by the measurement under different $\mathrm{pH}$ values. The results can be explained in the same way as those obtained by currentrectifying conical nanopores, although nanopipette-specific parameters should be taken into account for quantitative modeling. These current-rectifying nanopipette electrodes have a wide range of potential applications, including their biomedical use as multipurpose real-time in vivo biosensors.

\section{Preparation of Nanopipette Electrodes}

Experiments were performed with nanopipettes created as reported previously. ${ }^{11,16}$ Figure 1 is a cryo-SEM image of a noncoated, solution-filled nanopipette tip, showing the opening diameter of $\sim 55 \mathrm{~nm}$ in this particular case. PLL coating was achieved through a simple wetting with PLL aqueous solution, followed by a baking process for stabilization. The noncoated and PLL-coated nanopipettes were then implemented as electrodes for standard voltage clamp measurements, as shown in Figure $2 .{ }^{16}$ Potassium chloride $(\mathrm{KCl})$ was used as the electrolyte. The $\mathrm{KCl}$ concentration was set to $25 \mathrm{mM}$ for both the filling and bath solutions, and this ionic strength was maintained throughout the study.

\section{Current Response of Noncoated and PLL-Coated Nanopipettes}

To compare the current response of noncoated and PLL-coated nanopipettes, a 1-Hz alternating sine wave voltage with $\pm 1 \mathrm{~V}$ peak-to-peak amplitudes (Figure 3a) was applied to the working electrode inside the nanopipette under test. With noncoated nanopipettes, the current was suppressed during positive half-cycles (Figure $3 \mathrm{~b}$ ). This decrease in amplitude was consistently observed in tens of noncoated nanopipettes. With PLL-coated nanopipettes, however, the 
current was oppositely biased; that is, current was higher during positive half-cycles (Figure 3c). This tendency was reproduced with eight PLL-coated nanopipettes, while others showed less-pronounced but still high positive current compared with noncoated nanopipettes. The response with nonbaked PLL-coated nanopipettes was less stable than with baked nanopipettes, suggesting that direct electrostatic binding between the PLL and the quartz surface without free water molecules is required to obtain a stabilized response.

\section{Voltage Dependence of the Current Response}

Next we checked the difference between noncoated and PLL-coated nanopipettes over a wide range of applied voltages. A sine wave voltage sweep in $100 \mathrm{mV}$ steps was applied to investigate the input voltage dependence of the current response. The results of three examples are plotted on $I-V$ curves for noncoated (Figure 4a) and PLL-coated (Figure 4b) nanopipettes. Variations among three individual nanopipettes were seen for both conditions. Such variations have been recognized to be caused by differences in the nanopipette geometry. ${ }^{10}$ In addition, especially when coated with PLL, surface conditions may also contribute to the variability of the response. Future studies involving precise quantitative and statistical analysis of the observed amplitudes will require a normalization procedure.

The ratio of positive current peaks, $I_{+}$, to negative current peaks, $I_{-}$, at the same absolute voltage amplitudes seems suitable for quantifying the extent of rectification. ${ }^{17}$ We have introduced its logarithm, $r=\log _{2}\left(I_{+} / I_{-}\right)$, to equally deal with positive and negative rectification. Figure $4 \mathrm{c}$ shows $r$ values of nanopipettes from Figure $4 \mathrm{a}$ and $\mathrm{b}$. All three noncoated nanopipettes exhibited negative $r$ values similar to each other. PLL-coated nanopipettes, however, demonstrated variable $r$ values from zero to positive. This variability apparently derives from the variation of surface coverage of PLL on the nanopipette surface, which results in different net charge density. The data also shows that rectification was pronounced at higher applied voltages in both cases.

\section{Frequency Dependence of the Current Response}

The measurements above were all done at $1 \mathrm{~Hz}$. To determine the frequency dependence of the current response, a $\pm 1 \mathrm{~V}$ sine wave voltage between $1 \mathrm{~Hz}$ and $1 \mathrm{kHz}$ was applied. For both noncoated and PLL-coated nanopipettes, the response falls into two categories depending on the voltage frequency (Figure 5). At lower voltage frequencies $\left(10^{0}-10^{1} \mathrm{~Hz}\right)$, the current amplitude was asymmetric, that is, the current was rectified. Noncoated and PLL-coated nanopipettes rectified the current oppositely. At higher voltage frequencies $\left(10^{2}-10^{3} \mathrm{~Hz}\right)$, however, the output current amplitude was symmetric, that is, the current was no longer rectified. The same current amplitude was observed with noncoated and PLL-coated nanopipettes, and the amplitude itself correlated with the frequency in a proportional manner.

\section{Contribution of Surface Charge to the Observed Current Rectification}

The results above allow us to characterize noncoated and PLL-coated nanopipettes as negative and positive current rectifiers. It is considered to be caused by the inverted surface charge of the PLL coating. The surface of quartz nanopipettes is negatively charged because of the partial dissociation of the surface silanol groups in $\mathrm{pH}$-neutral solution, ${ }^{18}$ whereas the PLL layer makes this surface neutralized and further positively charged because of the protonated primary amine groups of the lysine residues. The contribution of surface charge can be experimentally confirmed by changing the solution $\mathrm{pH}$. The $\mathrm{KCl}$ solution without $\mathrm{pH}$ adjustment was slightly acidic $(\mathrm{pH}=5.0)$ in our condition. This solution $\mathrm{pH}$ was adjusted by the partial replacement of $\mathrm{KCl}$ with either $\mathrm{HCl}$ (giving acidic conditions that affect negative charges on the noncoated surface; Figure 6a) or $\mathrm{KOH}$ (giving basic conditions that affect positive charges on the PLLcoated surface; Figure 6b), and verified every time with commercially available test strips. 
Negative surface charge of noncoated nanopipettes should be neutralized at $\mathrm{pH}$ values as low as 2.0. ${ }^{19}$ When $\mathrm{pH} 2.0 \mathrm{KCl} / \mathrm{HCl}$ was used as both filling and bath solution, the $r$ values of a noncoated nanopipette approached zero (Figure 6a), which means a symmetric current response. This indicates that the negative rectification is indeed due to the negative charge on the quartz surface, as claimed previously. ${ }^{19}$ However, the replacement of only bath solution with lower $\mathrm{pH} \mathrm{KCl} / \mathrm{HCl}$ did not achieve fully symmetric current, possibly because of the imbalance of inner and outer $\mathrm{H}^{+}$concentrations that cannot be neglected at low $\mathrm{pH}$ conditions.

Similarly, increasing $\mathrm{pH}$ should affect PLL-coated nanopipettes. Although the state of coated PLL is hard to speculate, $\mathrm{pH} 9-11$ would be a good estimate for neutralization. Therefore, $\mathrm{pH}$ $12.0 \mathrm{KCl} / \mathrm{KOH}$ was tried for complete compensation of positive charges deriving from PLL. Interestingly, significant negative rectification was observed when a PLL nanopipette was simply dipped into $\mathrm{pH} 12.0$ bath solution (Figure $6 \mathrm{~b}$ ). This negative rectification was much more prominent than that in the case of a noncoated nanopipette in the same $\mathrm{pH}$ conditions.

\section{Explanation for the Observed Current Rectification}

Our results showed a current rectification effect with quartz nanopipettes, and its inversion upon PLL coating. The observed current rectification effect is consistent with, and supported by, preceding studies of ionic flow through nanopipettes ${ }^{12}$ or other hollow structures. ${ }^{14}, 17$, $20^{-} 23 \mathrm{In}$ addition, the inversion of rectification polarity has been confirmed in other nanoporous systems, including chemical modifications of gold-plated conical nanopores with amino-terminated thiols ${ }^{14}$ and charged macromolecular chains (DNA and polylysine), ${ }^{17}$ as well as silanization of silica nanotubes. 21 In these papers the modifications rendered the surface charge, resulting in the rectified current with opposite polarity. Despite differences in experimental details such as pore size, material, applied voltage, or ion concentrations, our results with quartz nanopipettes are generally in good agreement with these reports.

The similarity of experimentally observed results in similarly shaped nanostructures suggests that the mechanism underlying the observed phenomena is similar. Therefore, it is attractive to evaluate our own results in the existing context by applying well-established theories on nanofluidic ion transport phenomena. To begin with, we will briefly summarize the related notions proposed so far by the theoretical effort. ${ }^{10,12,14,20,21,24^{-29}}$ (1) The electric potential profile inside the pore governs the transport properties of the pore. (2) The electric potential can be largely influenced by the surface charge, and by the accompanying electrical double layer (EDL). (3) The presence of excess fixed charges can create the imbalance of the numbers of cations and anions in the lumen, resulting in the intrinsic permselectivity of the pore. (4) When an external voltage is applied, the electric potential profile is affected so that it induces the spatiotemporal change of ionic concentration profile, which explains the resulting currentvoltage relationship. (5) In the current rectification effect, asymmetry in the electric potential plays a significant role. These notions should be valid in any systems with similar nanostructures as long as the assumptions of the model are satisfied.

As a working model to explain our results, we chose a model proposed by Cervera et al.,24 originally intended for the explanation of current-rectifying conical nanopores. This model was also included in the recent review regarding the rectification in nanopores. ${ }^{30}$ The model with its assumptions and conditions seems to be best suited for our nanopipette system, especially because it covers a pore dimension larger than the Debye length. The nanopipette radius ( 25 $\mathrm{nm})$ and $\mathrm{KCl}$ concentration used $(25 \mathrm{mM})$ are within the assumptive range, and the relatively large opening radius should allow the use of continuum models. The only parameter in the model, the surface charge density of $-0.16 \mathrm{C} / \mathrm{m}^{2}$, is also comparable to ca. $-10^{-1} \mathrm{C} / \mathrm{m}^{2}$ for fused silica.25 Moreover, the presence of a long shank should not matter because the dominant part of the total resistance focuses at the pipette tip region (within a few micrometers of the 
tip) 16 where the shape is similar to that of the conical nanopore. Indeed, this geometric similarity has already been reflected in the electric field modeling of nanopipettes 10 and nanopores, 26 both independently showing a drastic, highly asymmetric change (i.e., a steep electric potential drop) at the proximity of the tip just inside the pore.

On the basis of these understandings, our results can now be linked to the Cervera model. Note that we intend to show the validity through the qualitative discussion in this Letter. First, the observed current rectification effect could be explained in the same way; that is, by the dynamic change of cation and anion concentrations inside the nanopipette. The enrichment or depletion of particular ions corresponds to the higher or lower current amplitudes, respectively. The consistency was further confirmed by the observation of the concentration dependence of rectification. The rectification effect appeared at or below $100 \mathrm{mM}$ but not at $1 \mathrm{M},{ }^{16}$ giving quantitative agreement with the prediction of the model; it predicts the selectivity of the pore to cations at $0.1 \mathrm{M}$ but not at $1 \mathrm{M}$ when the pore radius equals to $22 \mathrm{~nm}$ (Figure $5 \mathrm{~b}$ in ref 24). Therefore, the different concentrations and pore selectivity can be considered to underlie the current-rectifying nanopipettes.

Second, one can see that current rectification diminishes at high frequencies (Figure 5). Such high frequencies prevent the tip region from reaching its steady state as is assumed in the model; that is, the change of external voltage is too quick to achieve the formation of steady concentration profile at the tip that could represent a low or high electrical conductivity state. The inverse of the threshold frequencies around $10^{1}-10^{2} \mathrm{~Hz}$ may reflect the time required for full ionic translocation. ${ }^{20}$

Third, PLL coating inverted the direction of current rectification (Figure 4), and this was reinverted by the high $\mathrm{pH}$ condition (Figure 6). This can be explained by the change of the polarity of the surface charge. PLL makes the nanopipette surface positively charged at low and neutral $\mathrm{pH}$, changing the dominant ionic species at the narrow tip region from cations to anions. The high $\mathrm{pH}$ condition compensates for the positive charges of PLL, resulting in the recovery of cation-selective pore. Inverting the sign of the surface charge density in the model essentially leads to the exchange of concentration profiles between cations and anions, resulting in the current rectification with opposite polarity.

And last, with PLL-coated nanopipettes, we observed comparable or even more amplified current (Figure 4), and higher sensitivity to external $\mathrm{pH}$ change than with noncoated nanopipettes (Figure 6). Again, the surface charge density should be the key. The amplified current can be linked to the higher surface charge density that overcomes the effect of a physically narrowed path by the coated polymers. Such high charge density at the tip could result from the long, hydrophilic PLL chains. It is possible that these chains cause the sensitivity to $\mathrm{pH}$ change. Similar speculation has been proposed in the context of nanopores ${ }^{22}$ in which dangling PLL strands may be present and act as an open-close switch. ${ }^{17}$

Although it is possible to compare the qualitative features of the experiment with models, quantitative comparisons are more difficult for several reasons. A major reason is correctly determining the critical parameter, the surface charge density of the Cervera model. The factors that go into determining this parameter are the surface coverage of the PLL layer, electrokinetic processes such as the protonation and deprotonation of hydroxyl and amino groups ${ }^{21}$ under various conditions, and the estimation of the change in zeta potential (and the Debye length) due to the strong external electric field at the tip region. In addition to the surface charge density, building an optimized numerical model may require other nanopipette-specific parameters. The parameters of such a model should reflect differences in nanoscale geometry, material properties, and nanofluidic dynamics. Special attention should be paid to any factors that could differentiate the properties in nanospace from those in bulk. Despite these difficulties, 
overcoming these challenges will lead to a design for a nanopipette-based device that controls the ionic flow: an analogue of semiconductor devices. ${ }^{23,}, 31$

\section{Future Applications}

Understanding the nanopipette properties is an essential step in developing nanopipette biosensors, whose sensing zone is focused at the tip proximity. ${ }^{16}$ One direct application of this study could be the use of nanopipettes as $\mathrm{pH}$ nanosensors, which would take advantage of their $\mathrm{pH}$ dependence of electrical signals. By exploring the sensitivity to the changes of environmental $\mathrm{pH}$, one might be able ultimately to establish a nanopipette-based real-time in vivo $\mathrm{pH}$ sensor similar to the ion-sensitive microelectrodes, ${ }^{32}$ which does not require the introduction of optical indicators ${ }^{33}$ that could perturb the target cells. The ultrathin tip structure of a nanopipette is particulary suitable for the direct measurements inside single living cells. Targeting specific cellular compartments and intracellular organelles could unveil additional correlations between intracellular $\mathrm{pH}$ and cellular functions at a single cell level.

Observing the effect of PLL coating provides insight into additional modification of the nanopipette surface for other purposes. It may also be possible to monitor the functionalization by an amine-reactive reagent because it will affect the surface charge derived from PLL. Even biomolecule detection itself could be possible if the target molecules significantly affect surface conditions. This surface-condition-based detection can be used complementary to or together with current-blockade-based detection, which was already reported for nanoparticles passing through the nanopipette tip. ${ }^{11}$ That could open up new possibilities to sensitive detection of a wide variety of biomolecules in real time. Metabolite detection upon the exposure to drug candidates, either in vitro or in vivo, is currently within the reach of this technique.

In conclusion, we have shown that positively charged PLL coating has dramatically changed the ionic current response of quartz nanopipette electrodes to an identical applied voltage. An alternating sine wave voltage input resulted in rectified current in both noncoated and PLLcoated nanopipettes, but the current was amplified and rectified in an opposite direction with PLL-coated nanopipettes. The current rectification was observed with an applied voltage amplitude higher than $10^{-1} \mathrm{~V}$ and frequencies lower than $10^{1} \mathrm{~Hz}$ with dependence on the surface charge. The results were consistent enough with preceding studies using nanopipettes and conical nanopores so that the related theoretical approaches were directly applied to show a common underlying mechanism. The concentration-based explanation proposed by the nanopore community qualitatively validated our results, still leaving room for improvements by introducing nanopipette-specific quantitative parameters. The finding will not only deepen the understanding of physicochemical properties of current-rectifying nanostructure but will also be used as a basis for nanopipette-based in vivo biosensors.

\section{Supplementary Material}

Refer to Web version on PubMed Central for supplementary material.

\section{Acknowledgments}

This work was supported by NIH HG003448 and PO1-HG000205. We gratefully acknowledge the invaluable discussions with our collegues Henrik H. J. Persson and Jonathan S. Daniels. We are thankful to Dr. Hyonchol Kim for his support in obtaining SEM and cryo-SEM images. We thank Dr. Mathew W. Brock for discussing the practical side of nanopipette fabrication. S.U. and K.Y. acknowledge financial support from Japan Science and Technology Agency.

\section{References}

1. Rosi NL, Mirkin CA. Chem. Rev 2005;105:1547-1562. [PubMed: 15826019] 
2. Zanchet D, Micheel CM, Parak WJ, Gerion D, Alivisatos AP. Nano Lett 2001;1:32-35.

3. Patolsky F, Zheng G, Hayden O, Lakadamyali M, Zhuang X, Lieber CM. Proc. Natl. Acad. Sci. U.S.A 2004;101:14017-14022. [PubMed: 15365183]

4. Majumder M, Chopra N, Hinds BJ. J. Am. Chem. Soc 2005;127:9062-9070. [PubMed: 15969584]

5. Deamer DW, Akeson M. Trends Biotechnol 2000;18:147-151. [PubMed: 10740260]

6. Wadu-Mesthrige K, Amro NA, Garno JC, Xu S, Liu G. Biophys. J 2001;80:1891-1899. [PubMed: 11259301]

7. Hong MH, Kim KH, Bae J, Jhe W. Appl. Phys. Lett 2000;77:2604-2606.

8. Rodolfa KT, Bruckbauer A, Zhou D, Korchev YE, Klenerman D. Angew. Chem., Int. Ed 2005;44:6854-6859.

9. Bruckbauer A, Zhou DJ, Ying LM, Abell C, Klenerman D. Nano Lett 2004;4:1859-1862.

10. Ying L, White SS, Bruckbauer A, Meadows L, Korchev YE, Klenerman D. Biophys. J 2004;86:10181027. [PubMed: 14747337]

11. Karhanek M, Kemp JT, Pourmand N, Davis RW, Webb CD. Nano Lett 2005;5:403-407. [PubMed: 15794633]

12. Wei C, Bard AJ, Feldberg SW. Anal. Chem 1997;69:4627-4633.

13. Park CW, Knemeyer JP, Marme N, Moller M, Spatz J, Wolfrum J, Sauer M. Chem. Phys 2004;301:105-110.

14. Siwy Z, Heins E, Harrell CC, Kohli P, Martin CR. J. Am. Chem. Soc 2004;126:10850-10851. [PubMed: 15339163]

15. Li F, Chen Y, Sun P, Zhang MQ, Gao Z, Zhan DP, Shao YH. J. Phys. Chem. B 2004;108:3295-3302.

16. See the Supporting Information for details.

17. Harrell CC, Kohli P, Siwy Z, Martin CR. J. Am. Chem. Soc 2004;126:15646-15647. [PubMed: 15571378]

18. Senn BC, Pigram PJ, Liesegang J. Surf. Interface Anal 1999;27:835-839.

19. Parks GA. Chem. Rev 1965;65:177-198.

20. Siwy Z, Fulinski A. Phys. Rev. Lett 2002;89:198103. [PubMed: 12443155]

21. Fan R, Yue M, Karnik R, Majumdar A, Yang P. Phys. Rev. Lett 2005;95:086607. [PubMed: 16196887]

22. Apel PY, Korchev YE, Siwy Z, Spohr R, Yoshida M. Nucl. Instrum. Methods Phys. Res., Sect. B 2001;184:337-346.

23. Karnik R, Fan R, Yue M, Li D, Yang P, Majumdar A. Nano Lett 2005;5:943-948. [PubMed: 15884899]

24. Cervera J, Schiedt B, Neumann R, Mafé S, Ramírez P. J. Chem. Phys 2006;124:104706. [PubMed: 16542096]

25. Stein D, Kruithof M, Dekker C. Phys. Rev. Lett 2004;93:035901. [PubMed: 15323836]

26. Lee S, Zhang Y, White HS, Harrell CC, Martin CR. Anal. Chem 2004;76:6108-6115. [PubMed: 15481960]

27. Nishizawa M, Menon VP, Martin CR. Science 1995;268:700-702. [PubMed: 17832383]

28. Woermann D. Phys. Chem. Chem. Phys 2003;5:1853-1858.

29. Kosińska ID. J. Chem. Phys 2006;124:244707. [PubMed: 16821996]

30. Siwy Z. Adv. Funct. Mater 2006;16:735-746.

31. Daiguji H, Oka Y, Shirono K. Nano Lett 2005;5:2274-2280. [PubMed: 16277467]

32. Voipio, J.; Pasternack, M.; Macleod, K. Microelectrode Techniques: The Plymouth Workshop Handbook. 2nd ed. Ogden, DC., editor. Vol. Chapter 11. Cambridge, U.K: Company of Biologists; 1994. p. 448

33. Ohmichi T, Kawamoto Y, Wu P, Miyoshi D, Karimata H, Sugimoto N. Biochemistry 2005;44:71257130. [PubMed: 15882051] 


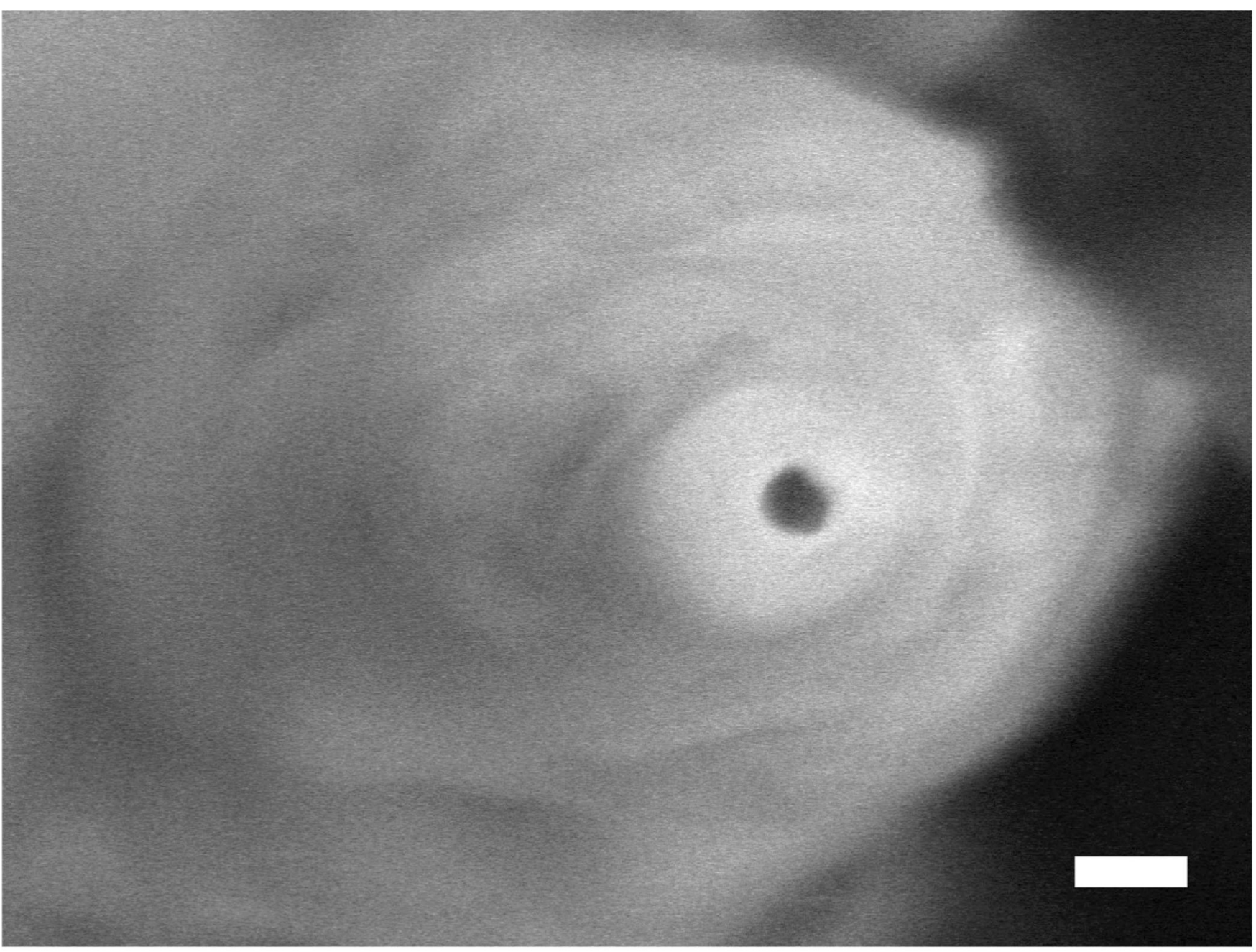

Figure 1.

Cryo-SEM image of a typical noncoated, solution-filled nanopipette tip (opening diameter = $\sim 55 \mathrm{~nm}$ ). Scale bar, $100 \mathrm{~nm}$. 


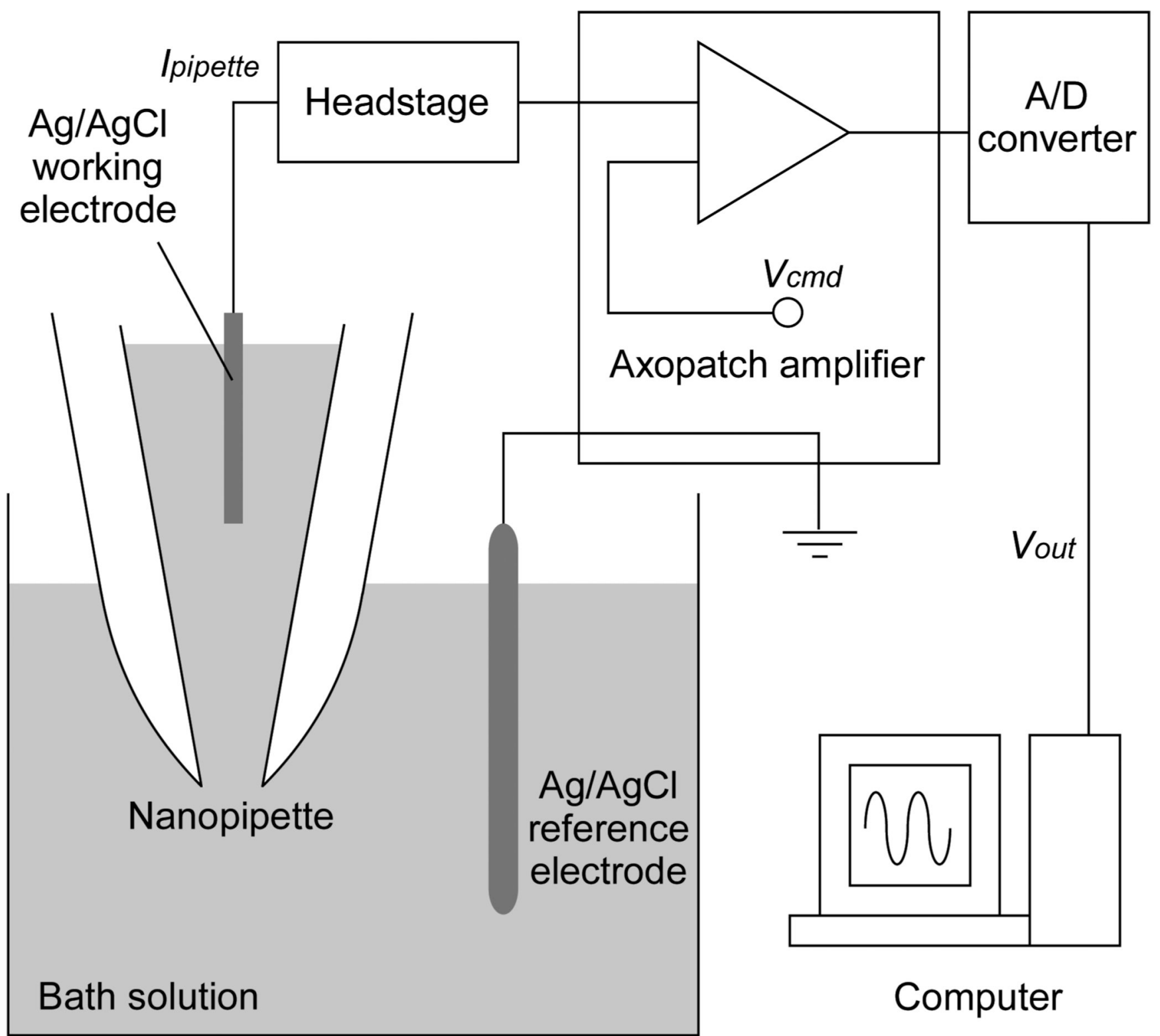

Figure 2.

Experimental setup. The electrolyte-filled nanopipette acts as a fine electrode. The current signal $\left(I_{\text {pipette }}\right)$ was converted to voltage output $\left(V_{\text {out }}\right)$ and sent to PC through the A/D converter at real time. The amplifier also allows the application of a specific voltage input $\left(V_{\mathrm{cmd}}\right)$ during measurement. 

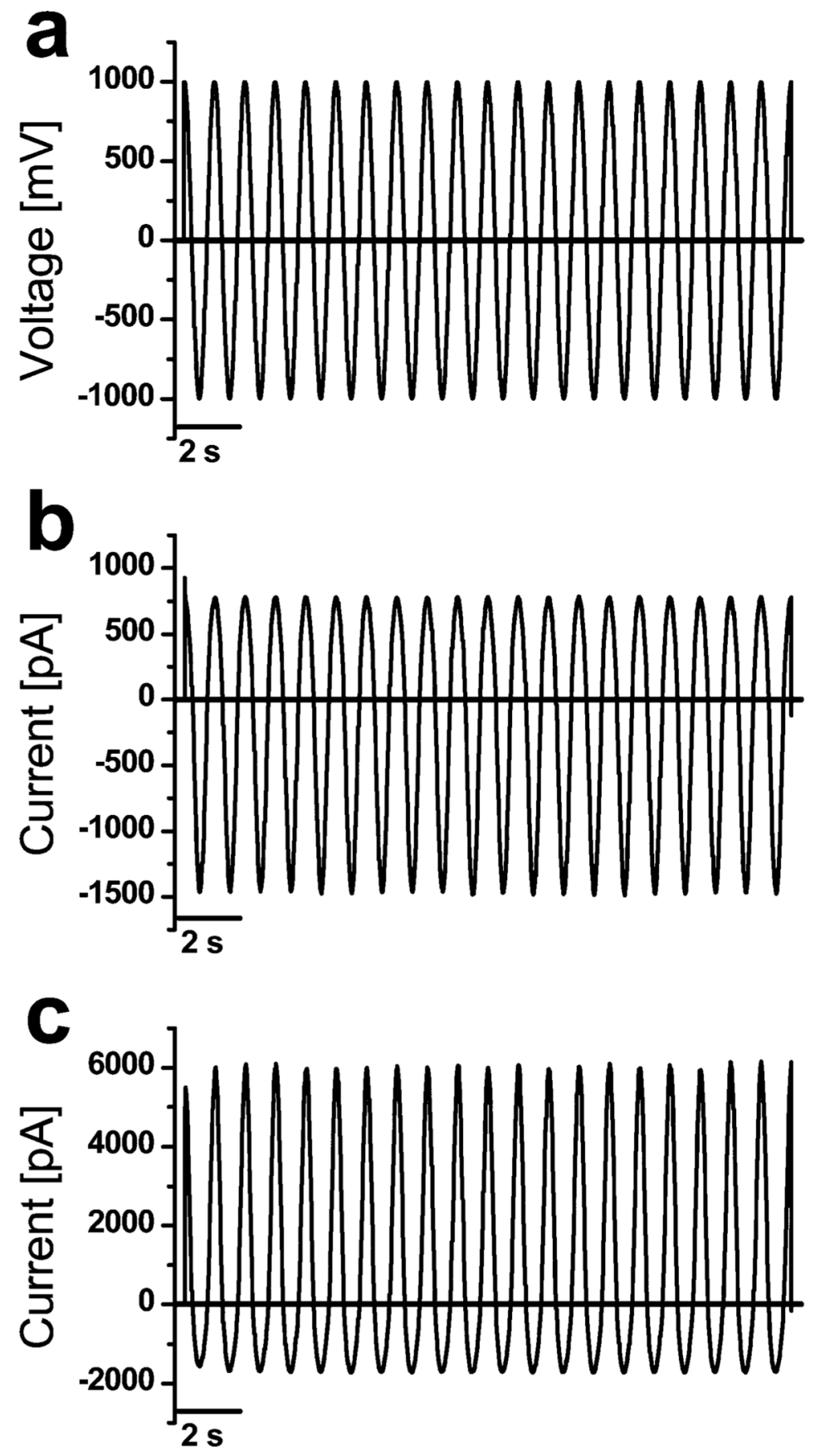

Figure 3.

Examples of the current response of noncoated and PLL-coated nanopipettes. (a) Applied 1 $\mathrm{Hz}$ sine wave voltage input. The amplitude and frequency was controlled in the following voltage- and frequency-dependence experiments. (b) Representative current response of a noncoated nanopipette. (c) Representative current response of a PLL-coated nanopipette. 


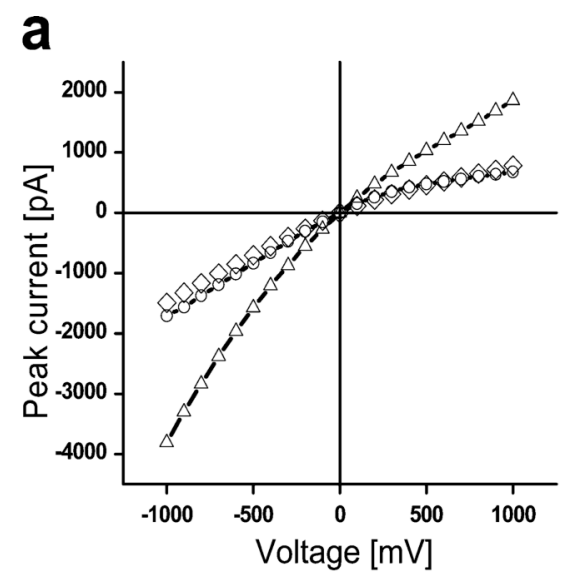

b

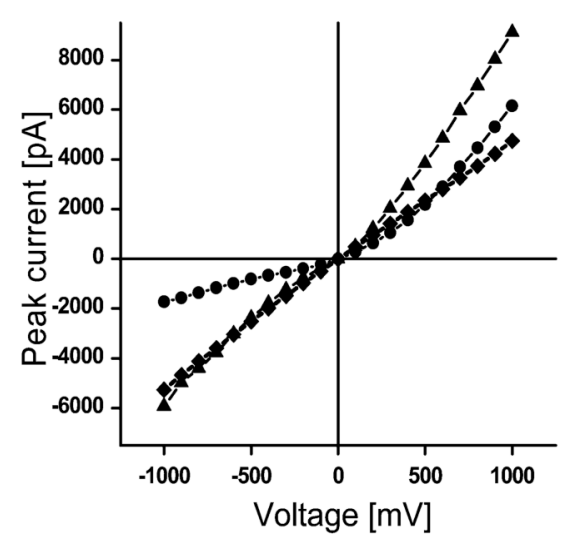

C

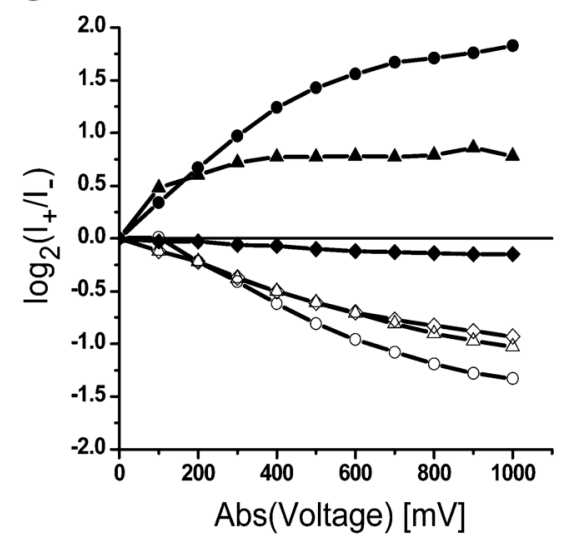

Figure 4.

Input voltage dependence of the current response. Current responses to $1 \mathrm{~Hz}$ sine wave voltage sweeps (with $100 \mathrm{mV}$ half-peak amplitude steps from $100 \mathrm{mV}$ up to $1 \mathrm{~V}$ ) were recorded. Each voltage sweep consists of a sine train of 10 or more periods. The sweep was analyzed to give the positive and negative peak current values to plot current-voltage ( $I-V)$ curves. (a) $I-V$ curves for three individual noncoated nanopipettes. (b) $I-V$ curves for three individual PLLcoated nanopipettes. (c) $r$ values (see text for definition of $r$ ) for the nanopipettes shown in a and $b$. Each symbol corresponds to the recorded data obtained with a particular nanopipette. 

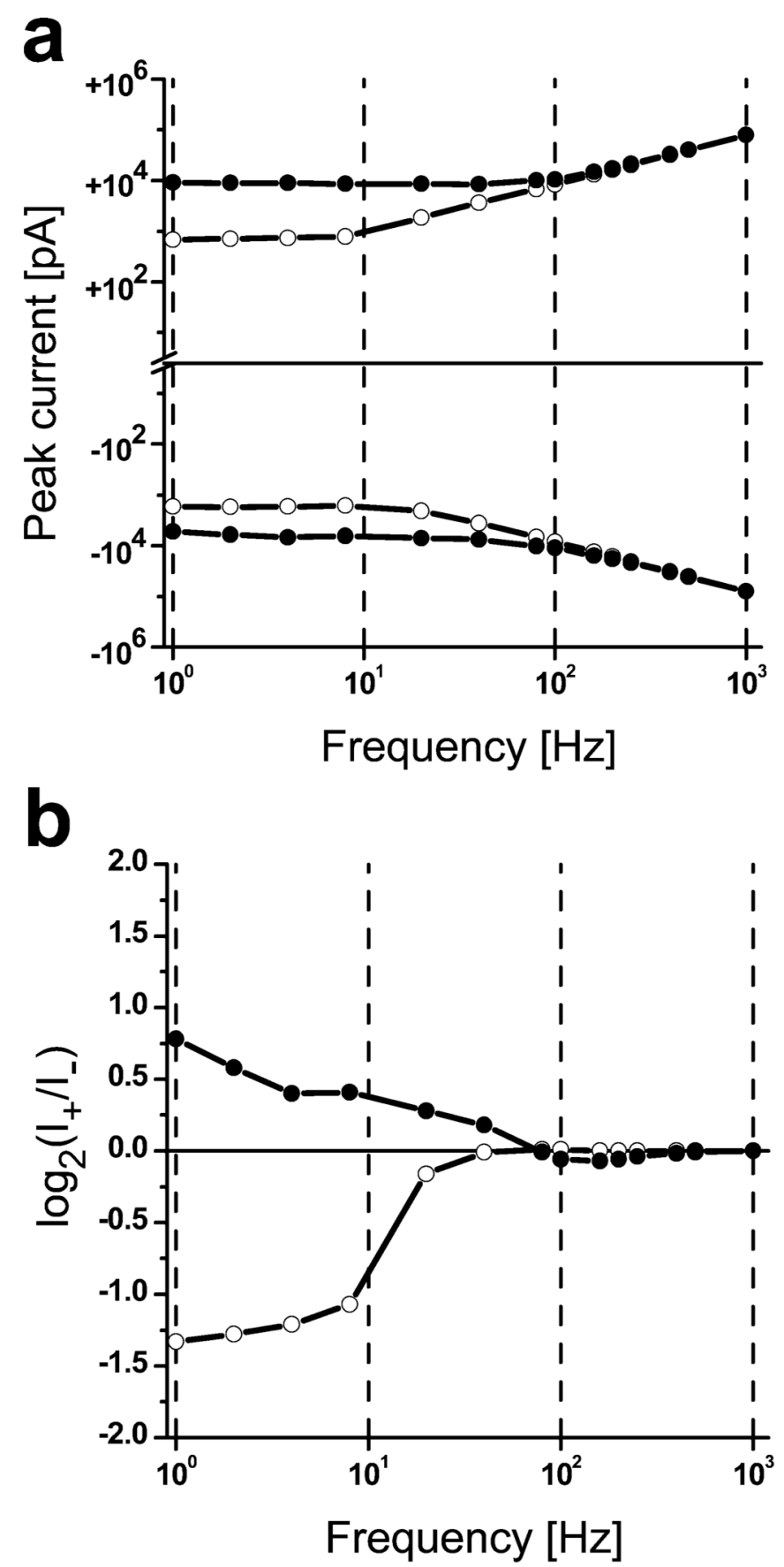

Figure 5.

Input frequency dependence of the current response. A $1 \mathrm{~V}$ half-peak sine wave at frequencies between $1 \mathrm{~Hz}$ and $1 \mathrm{kHz}$ was applied, and the corresponding positive and negative peak current values were recorded. (a) Current-frequency relationship of a noncoated (open triangles) and a PLL-coated (filled circles) nanopipette. Positive and negative peak current values are plotted symmetrically to clarify the tendency. (b) $r$ values for the nanopipettes shown in a. 

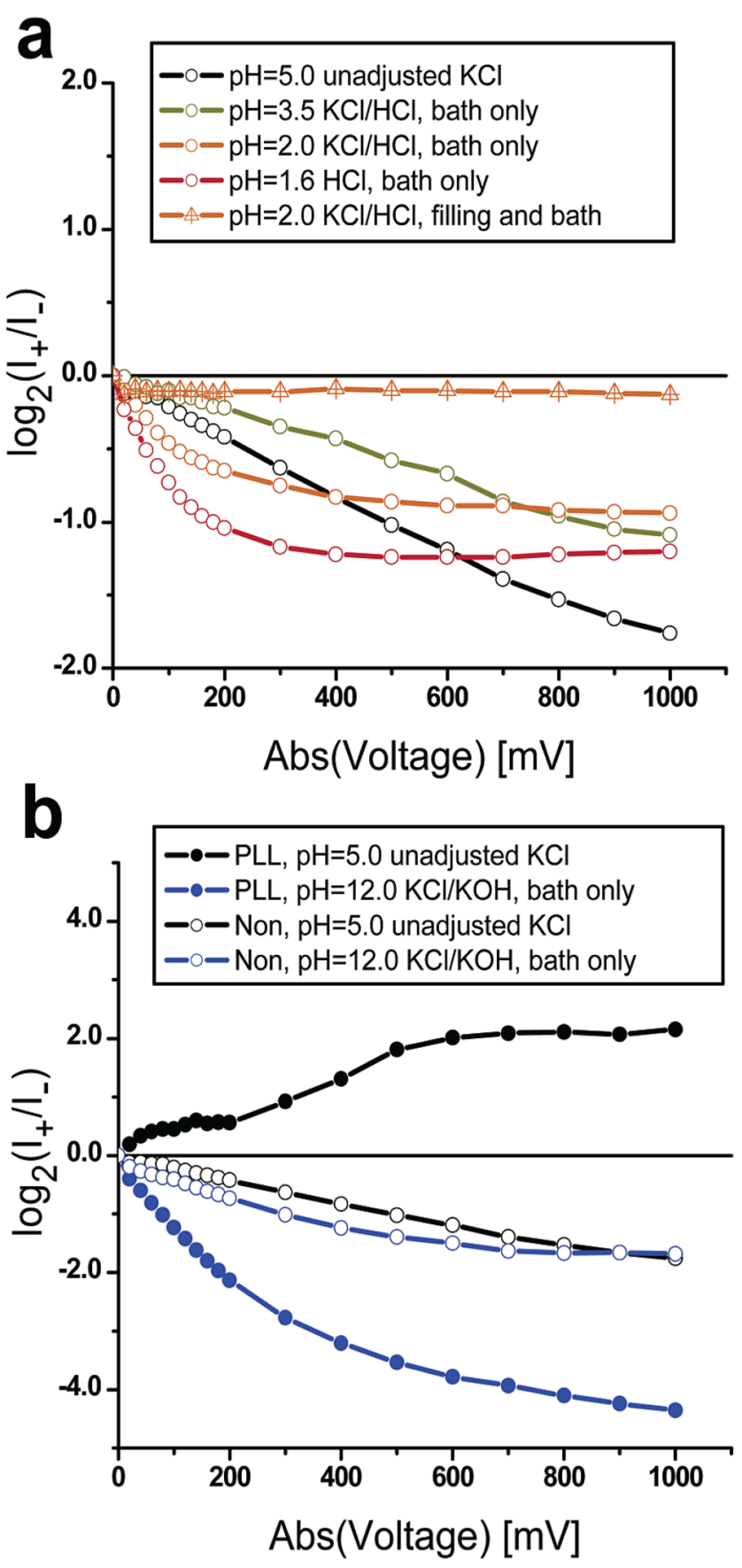

Figure 6.

$\mathrm{pH}$ dependence of the current rectification. (a) Surface charge neutralization of noncoated nanopipettes by acidic conditions. A noncoated nanopipette (open circles) was filled with $\mathrm{KCl}$ (without $\mathrm{pH}$ adjustment), measured in the same unadjusted $\mathrm{KCl}$ bath solution, and then sequentially dipped into other $\mathrm{KCl} / \mathrm{HCl}$ bath solutions adjusted to different $\mathrm{pH}$. Another noncoated nanopipette (signatured triangles) was filled with $\mathrm{pH} 2.0 \mathrm{KCl} / \mathrm{HCl}$ and measured in the same $\mathrm{pH} 2.0$ bath solution. (b) Surface charge inversion of PLL-coated nanopipettes by basic conditions. A PLL-coated nanopipette (filled circles) was filled with unadjusted $\mathrm{KCl}$ and measured in the same $\mathrm{KCl}$ bath solution, and then moved into a $\mathrm{pH} 12.0$ bath solution. The measurement with a noncoated nanopipette (open circles) followed the same procedure. 